\title{
A New Overview on the Old Topic: The Theoretical Analysis of “Combinatorial Strategy” for DNA Recombination
}

\author{
Gang Zhang ${ }^{1,2,3, *}$ \\ ${ }^{1}$ Department of Cell \& Systems Biology, University of Toronto, Toronto, Canada \\ ${ }^{2}$ Department of Medicine, Centre for Research in Neurodegenerative Diseases, University of Toronto, Toronto, Canada \\ ${ }^{3}$ Division of Nephrology, Massachusetts General Hospital, Harvard Medical School, Harvard University, Charlestown, United States \\ *Corresponding author: gang.zhang@utoronto.ca; sdzbzhanggang@yahoo.com
}

Received March 18, 2013; Revised October 24, 2013; Accepted November 15, 2013

\begin{abstract}
To clone genes of interest into suitable vectors is the first step to investigate their functions in vitro and in vivo. At the genome era, the sequences of more and more genes were decoded and available gradually. Therefore, it is critical to develop high efficient strategies for cloning genes of interest into different vectors to facilitate the functional analyses of them. In our previous studies, we created "Combinatorial strategy" for DNA recombination. Here, I theoretically analyzed the procedure of DNA recombination, the mechanism of this strategy, and further gave suggestions and predictions for various ligation-dependent molecular cloning experiments.
\end{abstract}

Keywords: combinatorial strategy, DNA recombination, clone sites, CIP, T4 DNA ligase, transformation, Top10

Cite This Article: Gang Zhang, "A New Overview on the Old Topic: The Theoretical Analysis of "Combinatorial Strategy” for DNA Recombination.” American Journal of Biomedical Research 1, no. 4 (2013): 108-111. doi: 10.12691/ajbr-1-4-6.

\section{Introduction}

The ligation-dependent DNA recombination technique was first developed at 1972 and 1973 [1,2]. From then on, the functions of genes of interest could be investigated intentionally in vitro and in vivo by cloning those of interest into appropriate vectors. In addition, the establishment of site-directed mutagenesis technique enabled the scientists to change the DNA sequences at specific locations [3]. These two techniques made the gene engineering and protein engineering from concepts into reality. During the past decade, the advent of the era of genomics unveiled the miracles of life codes unprecedentedly $[4,5,6]$. A main task of current biomedical research is to investigate the interactions of different proteins within the complicated networks, and the relationships of their functions. Thus far, more and more gene sequences of different organisms were gradually available, therefore, it is obvious that to efficiently clone appropriate vectors with genes of interest is the pivotal step for successful experimental investigations. At present, although several different strategies for molecular cloning were established, there were still no generalized methods to clone different vectors accurately and quantitatively with high efficiencies. In this review, based on our previous reports $[7,8]$, I theoretically analyzed our new method, "Combinatorial strategy", for efficient cloning different vectors.

\section{Analysis of the Procedure of DNA Recombination}

\subsection{What Happened in the Ligation Reaction System?}

In a typical 20 $\mu$ l ligation system, about 100 ng insert and $200 \mathrm{ng}$ vector DNA were mixed together with $1 \mu \mathrm{l} \mathrm{T} 4$ DNA ligase. Let us suppose that the sizes of the insert and vector are about $1.5 \mathrm{~kb}$ and $5 \mathrm{~kb}$, respectively. The average molar weight of A, G, C, T is about $660 \mathrm{~g}$. Therefore, 100 ng inserts contained about $3 \times 10^{10}$ insert DNA molecules, and $200 \mathrm{ng}$ vectors contained about $2 \times 10^{10}$ vector DNA molecules. So, how did these billions of insert and vector DNA molecules react with each other?

\subsection{Main Procedure of DNA Recombination}

Generally to speak, the main procedure of DNA recombination includes five steps. The first step is to choose and create compatible clone sites between the vectors and inserts. This is the starting point and critical for the successful cloning. The basic rule is to choose high efficient clone sites, such as EcoR I, BamH I, EcoR V, etc. both over-hang and blunt end sites, and to avoid using sites of low efficienies. The second step is to digest and purify the vector and insert DNA, and the value of the purities (A260/280) of all the DNAs must be above 1.80. At the ligation step, high concentration T4 DNA ligase 
should be used. And competent cells, such as DH5 $\alpha$, Top10 cells, could be used for transformation. Finally, 5 to 10 clones might be selected for identification by restriction digestion and confirmed by sequencing.

\subsection{Approaches to Create Compatible Clone Sites}

During the molecular cloning, the ideal circumstance was to find matching clone sites between the inserts and vectors among the multiple clone sites. Unfortunately, this is not always realistic in many molecular cloning experiments. In these cases, it was necessary to create the compatible clone sites between vectors and inserts. The mostly used method is to design PCR primers containing proper clone sites for the inserts. This is an easy and simple way to incorporate clone sites into the inserts, because both the 5' and 3' end clone sites could be designed and created in a single PCR reaction. The main disadvantage of this method is that the extra 3-4 bases at the terminal restriction sites are insufficient for stable association with and cut by certain restriction endonucleases $[9,10]$. In addition, because the PCR products of this method are linear DNA with incorporated clone sites, after restriction endonuclease digestion and agarose gel purification, theoretically, it could not guarantee that $100 \%$ of the digested DNA molecules were with correct cutting ends. Therefore, this method is only suitable for the small size, high efficient cloning, such as pET, pcDNA vectors, but might not for the large size, low efficient cloning, such as lentiviral vector cloning. Making blunt ends for the inserts and vectors is another method for molecular cloning. This method could neglect the differences between different over-hang clone sites. But in real experiments, this method is also of low efficiency due to two reasons. One is the low efficiency of blunt end ligation compared with cohesive ends. The other is the inefficiency of creation of correct blunt ends. The functions of Klenow fragment and T4 DNA polymerase are to make blunt ends by filling-in the 5'-overhangs, and removing the 3'-overhangs. At the same time, both of them could result in recessed ends due to their 3' to 5' exonuclease activity [11]. As a result, this method also could not guarantee that $100 \%$ of the products were with correct ends. Therefore, it is only suitable for high efficient cloning, but not for low efficient cloning. Site directed mutagenesis is a powerful tool to change the DNA sequences specifically [3]. It could be used to insert clone sites for molecular cloning [12]. The working format of the Stratagene SDM contained three steps. The first is to synthesize the mutated DNA strands by PCR. Then the methylated and hemimethylated parental DNA was digested by Dpn I enzyme. Finally, the mutated plasmids were transformed into the competent cells for multiplication. After growing bacteria and miniprep, the modified clone sites were created within the circular double-stranded plasmid DNA. Because the mutated products by SDM are circular double-stranded plasmid DNA, after digestion and purification, the linearized DNA is theoretically $100 \%$ with the correct-cutting ends. As a result, the maximal ligation could be achieved with the vectors, and the efficiencies of transformation could be improved radically. Therefore, this method is not only suitable for regular cloning, but also for low efficient cloning $[7,8]$.

\subsection{The Function of T4 DNA Ligase}

The function of DNA ligase is to catalyze the formation of 3', 5'-phosphodiester bond between the juxtaposed 5'phosphate and 3'-hydroxyl groups [13]. In vitro experiments using plasmid or synthetic oligonucleotide substrates revealed that T4 DNA ligase exhibited a relaxed specificity, 3' and 5' A-A or T-T mismatches, 5' G-T mismatches or 3' C-A, C-T, T-G, T-T, T-C, A-C, G-G or G-T mismatches $[14,15,16]$. Therefore, ligation could take place when there were mismatches at or close to the ligation junction by T4 DNA ligase. That is to say, between different clone sites, phosphodiester bond could be formed. This is the reason why there are lots of clones with empty vectors in molecular cloning experiments.

\subsection{Procedure of Regular Ligation}

There are three steps during regular ligation. One is the Inter-molecular reaction, at this step, to form noncovalently bonded, linear vector-insert hybrids, and this reaction requires high DNA concentrations. The other is the intra-molecular reaction, at that step, to form noncovalently bonded, circular molecules. That reaction works efficiently with low DNA concentrations. Finally, the annealing between inter and intra molecules brings the 5'-phosphate and 3'-hydroxyl residues of the vectors and inserts into close alignment, which allows T4 DNA ligase to catalyze the formation of 3', 5'-phosphodiester bond [17] (Figure 1). Because the ligation could take place between inter and intra molecules. At the same time, T4 DNA ligase could catalyze the ligation between different clone sites. Therefore, at least, there are three kinds of ligation can happen, which are the vector self-ligation, the insert self-ligation, and the vector-insert ligation. After transformation, all the circular DNAs could be efficiently transformed into the competent cells [2]. Because the vectors contain antibiotic resistance genes, the clones with vectors and recombinants can survive after antibiotic selection. Whereas, the clones only with circular inserts cannot survive the selection. Therefore, there are many background clones with empty circularized vectors (Figure 2).

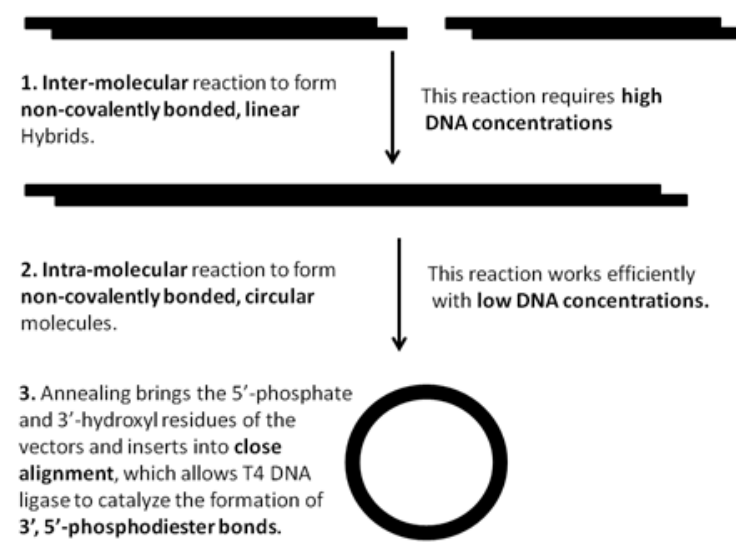

Figure 1. The procedure of regular ligation (modified from Sambrook J, Russell DW. 2001. Molecular Cloning: A Laboratory Manual) 


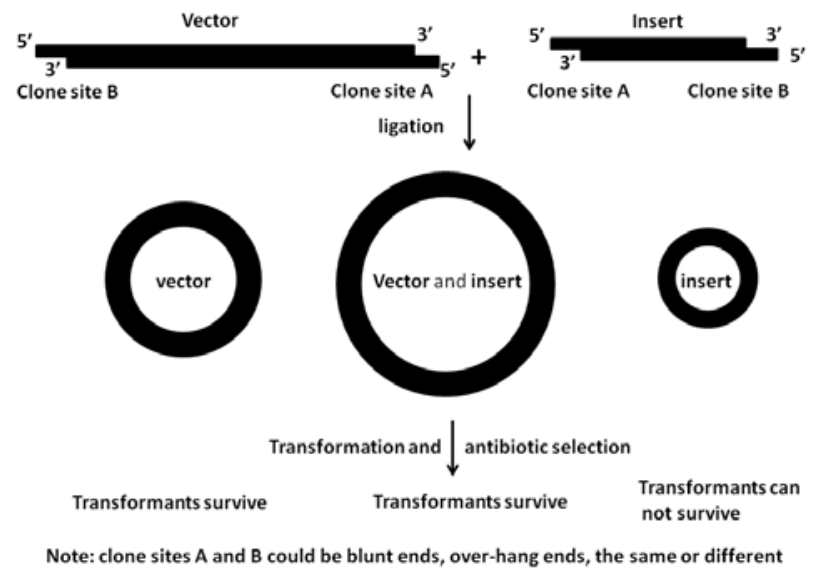

Figure 2. Types of ligation and transformation

\section{Working Mechanism of "Combinatorial Strategy"}

\subsection{The Function of CIP Treatment of the Vector after Enzyme Digestion}

The function of calf intestinal phosphatase (CIP) is to remove the 5'-phosphate group from the vector DNA [17]. After CIP treatment, the vectors could not be self-ligated. Because the transformation efficiencies of linear DNA are much lower than circularized DNA [2], the backgrounds with empty-vectors are decreased radically (Figure 3). Almost all the clones are with recombinant vectors $[7,8]$.

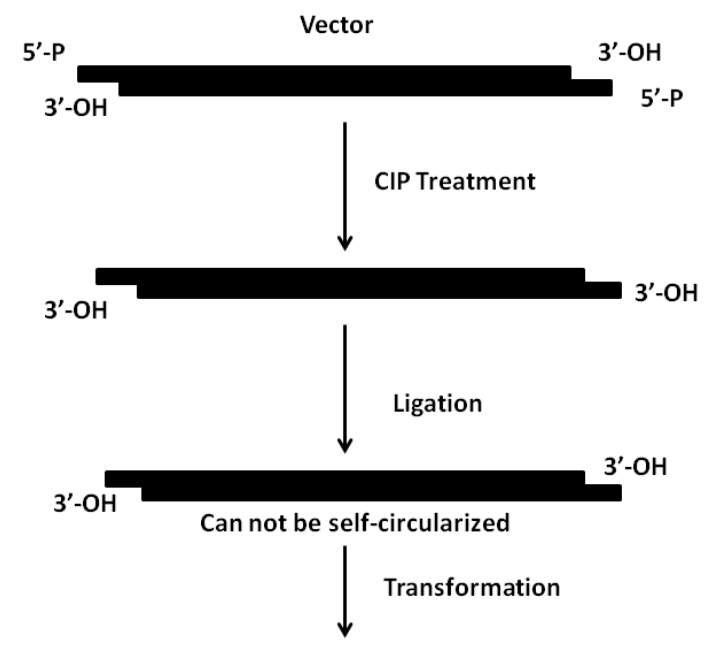

Because the transformation efficiencies of linear DNA are very low, the backgrounds with empty-vectors are decreased radically.

Figure 3. Function of CIP treatment (modified from Sambrook J, Russell DW. 2001. Molecular Cloning: A Laboratory Manual)

\subsection{Choosing Proper Competent Cells for Transformation}

After CIP treatment, the ligation efficiencies between the vectors and inserts are decreased significantly [17]. To increase the transformation efficiencies, more efficient competent cells should be used. DH5 $\alpha$ and Top10 competent cells are commonly used for transformation, because the transformation efficiency of Top10 cells $\left(1 \mathrm{X} 10^{9} \mathrm{cfu} / \mu \mathrm{g}\right.$ super coil DNA, Life Technologies) is much higher than DH5 $\alpha\left(1 \times 10^{6} \mathrm{cfu} / \mu \mathrm{g}\right.$ super coil DNA,
Life Technologies), Top10 cells are recommended as transformation hosts for CIP-treated ligation, in order to obtain sufficient clones for identification, particularly for CIP-treated, blunt-end cloning $[7,8]$.

\subsection{Theoretical Analysis of Combinatorial Strategy}

From the above analysis, we could conclude that, first of all, digesting the inserts from circular vectors could guarantee that $100 \%$ of the linearized inserts were with correct cutting ends. Secondly, the vectors treated with CIP could efficiently protect the vector-self-ligation, and decrease the background clones with empty-vectors. Finally, the clones with circular inserts could not survive the selection. And the transformation efficiencies of the linear vectors were very low. Therefore, most of the clones should be with the recombinant vectors. We could predict that, with different clone sites, nearly $100 \%$ of the clones should be the positive clones. With the same overhang, and blunt clone sites, about $50 \%$ of the clones should be the positive clones [7,8] (Figure 4).

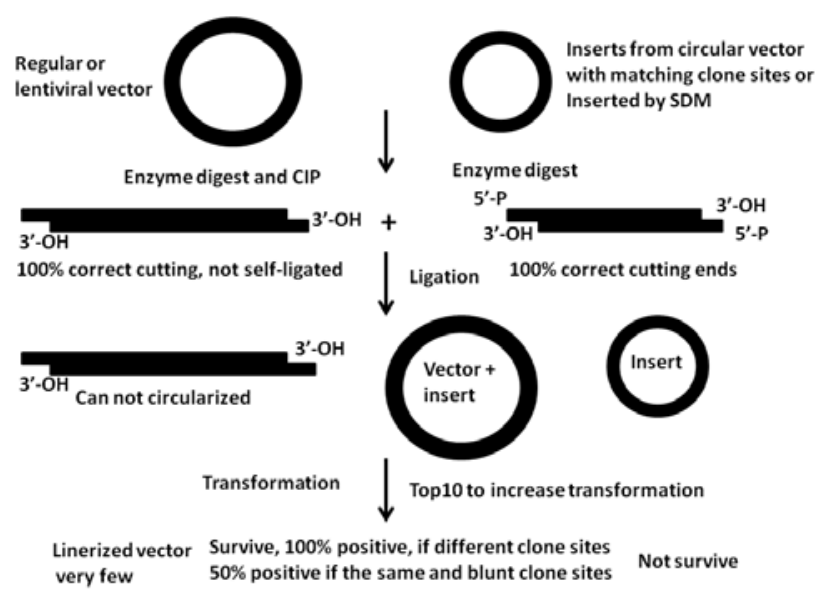

Figure 4. Working mechanism of "Combinatorial Strategy"

\section{Suggestions and Predictions for Molecular Cloning with CIP-Treated Vectors}

Our previous reports demonstrated that, with our "Combinatorial strategy", different plasmid vectors could be efficiently cloned with various clone sites [7,8]. Based on these studies, we could make the following suggestions and predictions for molecular cloning (Table 1). These suggestions could significantly facilitate the construction of vectors with genes of interest, and further speed up the biomedical research radically.

\section{Competing Interests}

The author declares that there is no competing interest.

\section{Acknowledgement}

The Parkinson Society of Canada (The Margaret Galloway Basic Research Fellowship 2005-2007 to G. Z.) and the Canadian Institutes of Health Research (CIHR, Grant MOP84501 to A. T.) financially supported this work. 
Table 1. Suggestions and predictions for molecular cloning with CIP-treated vectors

\begin{tabular}{|c|c|c|c|c|c|}
\hline Clone sites & Sizes (kb) & $\begin{array}{l}\text { Methods for clone } \\
\text { sites }\end{array}$ & $\begin{array}{c}\text { Transformation } \\
\text { host }\end{array}$ & No. of colonies & Positive clones \\
\hline \multirow{2}{*}{ Blunt sites } & Small (vector $<5$, insert<1.5) & Existed & Top10 & Dozens or more & About $50 \%$ \\
\hline & large (vector $>5$, insert $>1.5$ ) & Existed & Top10 & A few to dozens & About $50 \%$ \\
\hline \multirow{2}{*}{$\begin{array}{l}\text { Different over- } \\
\text { hang sites }\end{array}$} & Small (vector $<5$, insert $<1.5$ ) & SDM & Top10 & Hundreds or more & Nearly $100 \%$ \\
\hline & Large (vector>5, insert>1.5 ) & SDM & Top10 & Dozens to hundreds & Nearly $100 \%$ \\
\hline \multirow{2}{*}{ One over-hang site } & Small (vector $<5$, insert $<1.5$ ) & SDM & Top10 & Hundreds or more & About $50 \%$ \\
\hline & Large (vector $>5$, insert $>1.5$ ) & SDM & Top10 & Dozens to hundreds & About $50 \%$ \\
\hline
\end{tabular}

[8] Zhang, G. and Tandon, A., Combinatorial strategy: A highly efficient method for cloning different vectors with various clone sites, In submission, 2013.

\section{References}

[1] Jackson, D.A., Symons, R.H. and Berg, P., Biochemical method for inserting new genetic information into DNA of Simian Virus 40: Circular SV40 DNA molecules containing Lambda phage genes and the galactose operon of Escherichia Coli, Proc Nat Acad Sci USA, 69: 2904-2909, 1972.

[2] Cohen, S.N., Chang, A.C.Y., Boyer, H.W. and Helling, R.B., Construction of biologically functional bacteria plasmids in vitro, Proc Nat Acad Sci USA, 70: 3240-3244, 1973.

[3] Hutchison, C.A., Phillips, S., Edgell, M.H., Gillam, S., Jahnke, P. and Smith, M., Mutagenesis at a specific position in a DNA sequence, J Biol Chem, 253: 6551-6560, 1978.

[4] Adams, M.D., Celniker, S.E., Holt, R.A., et al., The geneome sequence of Drosophila Melanogaster, Science, 287: 2181-2195, 2000.

[5] Mardis, E.R., Next-generation DNA sequencing methods. Annu Rev Genomics Hum Genet, 9: 387-402, 2008.

[6] Mayer, A.N., Dimmock, D.P., Arca, M.J., Bick, D.P., Verbsky, J.W., Worthey, E.A., Jacob, H.J. and Margolis, D.A., A timely arrival for genomic medicine, Genet Med, 13: 195-196, 2011.

[7] Zhang, G. and Tandon, A., Quantitative assessment on the cloning efficiencies of lentiviral transfer vectors with a unique clone site, Sci Rep, 2: 415, 2012.
[9] Jung, V., Pestka, S.B. and Pestka, S., Efficient cloning of PCR generated DNA containing terminal restriction endonuclease recognition sites, Nucl Acids Res, 18: 6156, 1990.

[10] Testori, A., Listowsky, I. and Sollitti, P., Direct cloning of unmodified PCR products by exploiting an engineered restriction site, Gene, 143: 151-152, 1994.

[11] Sambrook, J., Fritsch, E.F. and Maniatis, T., Molecular Cloning: A Laboratory Manual, Cold Spring Harbor Laboratory Press: Plainview, NY, 1989.

[12] Norrander, J., Kempe, T. and Messing, J., Construction of improved M13 vectors using oligodeoxynucleotide-directed mutagenesis, Gene, 26: 101-106, 1983.

[13] Lehnman, I.R., DNA ligase: structure, mechanism and function. Science, 186: 790-797, 1974.

[14] Wu, D.Y. and Wallace, R.B., Specificity of the nick-closing activity of bacteriophage T4 DNA ligase, Gene, 76: 245-254, 1989.

[15] Harada, K. and Orgel, L., Unexpected substrate specificity of T4 DNA ligase revealed by in vitro selection, Nucl Acids Res, 21: 2287-2291, 1993.

[16] Landegren, U., Kaiser, R., Sanders, J. and Hood, L., A ligasemediated gene detection technique, Science, 241: 1077-1080, 1988.

[17] Sambrook, J. and Russell, D.W., Molecular Cloning: A Laboratory Manual, Cold Spring Harbor Laboratory Press: Plainview, NY, 2001. 